\title{
The Application of Desktop Virtualization Technique in College Computer Room Management
}

\author{
Shijie Wu \\ Tianjin Maritime College, Tianjin, China, 300350
}

\begin{abstract}
Keywords: Virtualization; Cloud server; Computer room management; Desktop virtualization technique
\end{abstract}

\begin{abstract}
As the development of information technology, Virtualization desktop technology has occupied a place in the management of the university computer room. Computer room management involves more content, virtual desktop technology can play a specific effect at different levels. This paper mainly explains the virtual desktop technology in the management of computer room in specific application, through the elaboration of virtual desktop technology framework, a detailed analysis of the application of desktop virtualization technology, maximum summed up the virtual desktop technology advantages and application effect in the management of computer room.
\end{abstract}

\section{虚拟化桌面技术在高校机房管理中的应用}

\author{
吴士杰 \\ 天津海运职业学院 中国 天津市 300350
}

摘要：随着当今社会信息科技的进步, 虚拟化桌面技术已经在高校机房管理中占据了一席之 地。机房管理涉及的内容较多, 虚拟化桌面技术能够在不同层面发挥出具体效果。本文主要 阐释虚拟化桌面技术在高校机房管理中的具体应用，通过对虚拟化桌面技术的框架结构的阐 述, 详细分析了虚拟化桌面技术的应用, 最大限度上总结出虚拟化桌面技术的优势以及在机 房管理中的应用效果。

关键词：虚拟化；云服务器；机房管理；虚拟化桌面技术

\section{1. 引言}

当前, 很多高校都采用计算机授课的模式, 这种授课模式能够培养学生的动手实践能力和创 新应用的能力。因此, 实验性教学是实现职业教育发展中的重要手段和方法。目前, 很多课 程采用的都是多样化教学, 那么机房除了要满足多样化的教学要求, 还要配置多样性的教学 环境。虚拟化桌面技术从根本上解决了传统机房的设备分散的老问题，使机房管理人员的管 理维护工作更加便捷和多样化，能够更加全面有效的维护和管理实践性教学。

\section{2. 虚拟化技术}

2.1. 虚拟化技术概述

虚拟化技术是一种基于中心服务器的计算机模型, 它是广义的说法。虚拟化技术是对物理事 物的抽象化描述, 从逻辑硬件中抽象出计算机的下层构件和应用程序, 这是从逻辑的角度进 行物理资源分配的较常用的手段。例如, 当前只有一台计算机, 我们运用虚拟化技术以后, 用户看起来就形成了多台计算机，而且每一台计算机都有自己的内存、硬盘、CPU 等硬件。 虚拟化技术实际上是实现了计算机的硬件和软件的分离, 这样只要在虚拟层上运行相应的软 件系统, 并不需要考虑硬件条件。

2.2. 虚拟化桌面技术

虚拟化桌面技术旨在改造虚拟化的桌面软件, 在中央服务器上改造出各种虚拟桌面分配给用 
户，提高了用户使用虚拟化桌面软件的效率。虚拟化桌面的主要包含两大技术，一是使用网 络服务器运行的虚拟化桌面, 尤其是 Google 的 Office 软件, 整个桌面能够浏览器里实现运 行, 程序的实现即是对原有桌面软件的重写过程。二是有效减少软件许可使用, 如果客户需 要软件就利用应用层虚拟化的帮助来提供桌面虚拟化, 用打包的方式将软件传送给用户。

\section{3. 虚拟化桌面技术架构}

虚拟化桌面技术架构 (Virtual Desktop Infrastructure, 简称 VDI) 是许多机构目前正在评 估的全新模式, 它基于初期的 RDP 协议和瘦客户机慢慢转变而来, 同时是 VMware 等国外虚拟 化商家一直推荐的模式。虚拟化桌面技术架构的最大特征是提升智能分布式计算的响应能力 和用户体验的定制化服务, 并能够集中化管理整个系统桌面。桌面虚拟化技术框架主要包括 四部分。

(1) 客户端。虚拟化桌面技术可以运行在任意的低版本电脑上, 只要打开客户端的虚拟应用 即可操作, 这样可延长计算机的使用时间, 从而降低学生学习成本的耗损。

(2) 服务器。具有数据备份、应用部署、资源管理和分配的辅佐功能, 主要对桌面的运行管 理和桌面操作系统的虚拟化起重要作用。

(3) 虚拟化平台。虚拟化平台能将客户端的桌面系统看成映射的镜像文件, 制作成一张虚拟 盘, 直接存在平台服务器中, 根据网络环境和需求提供比较完善的桌面系统服务。

(4) 存储。将集中管理、横向扩展、虚拟化存储等操作存储在专用的存储设备中, 并具有扩 展灵活、管理弹性、可靠性高等特征。

\section{3. 机房管理中的问题}

高校机房在使用过程中会出现一些问题, 机房使用率的不断提高, 必定会使问题更加突出, 解决问题的关键是找出原因。

\section{1 . 管理人员的问题}

高校机房管理人员担负着较大的日常工作量, 除了防护机房实验管理及环境安全工作的同时, 还要排查机房的技术维护和网络故障的工作。工作难度大、覆盖面广、事务较杂乱。每个机 房的计算机硬件和软件配置不统一, 安装软件的版本不统一, 这就要求机房管理人员必须要 有较强的计算机维修能力, 能够在短时间内排除故障。机房管理工作交复杂、繁琐, 尤其存 在着不同程度的技术难关。

\section{2. 实验环境的问题}

高校机房一般有较高使用率等特点, 因此, 机房环境卫生及其安全使用的维护较复杂。个别 学生上机时暴力操作, 导致设备损坏, 随意拔插电源、触碰电线引发事故, 随意安装软件及 接入储存设备, 造成计算机感染病毒, 导致实验设备及网络环境不能安全有效的使用, 严重 影响了实验教学任务。

\section{3 . 实验设备的损耗}

计算机设备的安放对其周围环境的要求非常高, 因此机房的场地选择、供配电、空调、电磁 波防护等方面提出要求。同时需要注意防火、防水、防静电、防雷击、防辐射、报警及消防 设施等, 这些都是保证计算机设备安全的硬环境。此外, 计算机也有自己的寿命, 部分硬件 设备如主板、显示器等, 也会因自然原因而损耗, 这些都影响了计算机的正常使用。

3. 4 . 设备维护的问题

有些高校机房建设、维护保养的资金投入较少, 计算机设备得不到定期的维护及保养。这样 导致机房无法达到正常使用的安全标准, 电源短路、设备老化等一系列的问题随之而来。这 些问题的出现既威胁了师生们的人身安全, 也影响了实验设备的安全使用。

\section{4. 虚拟化桌面技术的优势}

虚拟化桌面技术在机房管理方面的应用尤为突出, 它能有效提高管理质量和效率, 大幅缩减 
管理耗材成本，更在高效节能方面发挥了较大作用。

\section{1. 管理维护}

虚拟化桌面技术的全面应用, 使机房管理工作实现集中管理桌面环境、弹性分配硬件资源, 以及终端无需维护等方面的便利。机房管理人员可登陆服务器管理平台进行访问, 并能管理 及维护所有的服务器和桌面。网络下的客户端都集中在服务器端运行、存储程序和数据, 通 过这种新型工作模式, 机房管理人员可以快速地完成信息系统的安装、调试、升级和维护工作, 大大节省信息系统的部署时间，降低人力资源费用和管理成本，提高管理效率。

\section{2. 安全性能}

在虚拟化桌面技术环境下的客户端通常使用集成化的系统结构, 为了防止病毒来源, 没有软 驱、光驱、硬盘等配置, 进而使系统的可靠性和稳定性得到大幅提升。客户端的本地系统软件 采用嵌入式技术设计, 因此客户端从硬件和软件两方面都保证了系统的稳定运行, 其故障率 较之 PC 大大降低。因为客户端没有软驱、光驱、硬盘等配置, 就杜绝了用户将外来软件安装 或复制到系统中, 如此就不能随意复制重要软件和涉密数据, 从而有效保护了系统安全和信 息安全。

\section{3. 缩减成本}

缩减成本主要体现在采购和维护成本两方面。

虚拟化桌面技术, 客户端主要采用云终端的方式开展架构, 这样大大降低了计算机设备的采 购成本。此外, 云终端设备具有非常少的可移动部件, 所以它的使用寿命远远要高于传统 PC 机。

维护成本主要是维护桌面应用和软件授权等方面工作。对于桌面应用维护而言, 机房涉及的 设备数量较多, 机房管理员需要对不同的 PC 机安装操作系统及应用程序, 需要消耗的精力及 时间较大。如果使用虚拟化桌面技术, 那么机房管理人员则只需在服务器端进行一次操作就 能实现对整个机房的应用, 大大缩短了操作时间和精力。在软件授权方面, 大部分计算机上 运行的都不是正版软件, 这非常不利于软件行业的发展和知识产权的保护。而虚拟化服务器 上的软件必须使用正版软件, 这样客户端就不需要购买或安装任何软件, 同时为正版软件使 用者降低了使用成本，进一步促进了整个行业的迅猛发展。

4. 4. 节能环保

节能效应在机房管理过程中至关重要, 传统的 PC 机消耗功率通常在 250 瓦以上, 云终端由于 没有风扇, 功耗较低, 约 23 瓦, 使噪声污染和能源消耗大大减低。

\section{5. 虚拟化桌面技术在机房管理方面的应用}

5. 1 . 实验环境的部署

在虚拟化桌面的环境下, 客户端是以文件形式所存在的, 它和系统配置是无关的, 能够快速 的实现一个统一的实验环境。如果要对机房进行环境的部署就要先把实验所需要的系统环境 安装配置在虚拟化的平台中。这样就可以把客户端文件复制到任意一台安装有虚拟化平台的 计算机上直接使用了。它不会受系统环境的限制, 也不会受硬件配置的限制, 还能够有效地 把实验环境统一起来。根据机房教师机中的保护卡分区保护功能把装好的母盘直接传输给机 房中的学生机。

5.2. 多系统运行

一般情况下, 机房内的计算机都是性能比较单一的设备, 它的弊端在于需要多个操作系统或 者多台计算机同时进行, 就会出现由于负载导致服务器的失衡, 或者数据库的异地备份等情 况。特殊的技术就可以让一台计算机同时模拟多个系统环境, 每个系统都是独立的, 但又是 相辅相成的。

\section{3. 创建系统}

为了提高计算机的工作效率, 通常在计算机中要几个系统同时工作, 这样做是很浪费时间的。 所以一种更有效的方法是在虚拟化的环境下安装一个操作系统的母盘, 然后通过复制虚拟机 
的方式创建新系统。虚拟机的优点在于不会改变母盘的数据, 只会保存操作而产生的差异数 据。如此就成为更加高效和高兼容性的操作系统。

\section{6. 结束语}

虚拟化技术做为一项先进的教学实践手段较为科学，且新颖。它通过对真实世界的虚拟、模 仿, 实现实际设备上处理较为繁杂的项目。在机房管理中, 虚拟化技术还存在一些问题, 需 要我们进一步的研究分析。相信随着科技的不断发展, 虚拟化技术会越来越成熟, 它不仅可 以使机房管理更加高效、有序，也可以激发出学生们对计算机的学习积极性。随着虚拟化技 术的不断发展，虚拟化桌面技术必定会大规模地部署和应用在高等院校的机房和实验室中， 使计算机得到有效的管理、维护和运行, 促使机房管理员能从大量的管理维护工作中解放出 来，从而有效地提升机房管理工作的效率。

\section{参考文献}

[1] 崔一峰.虚拟化技术在高职院校计算机实践教学中的应用 $[\mathrm{J}]$.福建电脑.2012.05

[2] 罗杰,宁天桥.服务器虚拟化技术在机房的应用[J].计算机时代.2012.1

[3] 邱科宁.桌面虚拟化技术在机房管理中的应用研究 [J].福建电脑.2012 年第 1 期

[4] 阮建华.桌面虚拟技术在高校实验机房中的应用 [J].福建商业高等专科学校学报.2012 年第 2 期

[5] 刘卫宁.面向多任务的制造云服务组合[J].计算机集成制造系统，2013,19(1):199-209.

[6] 刘正朋.桌面虚拟化技术在实验室仪器管理中的应用[J].实验室科学, 2011,10(1):151一 153 .

[7] 韩芳, 袁宇宾.计算机桌面虚拟化技术在教学及管理中的实现路径研究[J]. 重庆理工大学学 报:自然科学,2014,10(7):105-109.

[8] 林曼.桌面虚拟化技术 $(\mathrm{VDI})$ 在高校实验室教学中应用研究 [J]. 计算机光盘软件与应 用,2013,2(18):205-206.

[9] 2013 地平线报告:信息技术挑战催生高等教育变革[EB/OL].

[10] 刘知云.高职计算机教学应用互助教学模式效果探析[J].考试周刊.2015

\section{References}

[1] Yifeng Cui. The Application of Virtualization Technique in Practical Teaching of University [J]. Fujian Computer. 2012.05

[2] Jie Luo, Tianqiao Ning. The Application of Server Virtualization Technology in Computer Room [J]. Computer Time.2012.01

[3] Kening Qiu. The Research of Desktop Virtualization Technique in Computer Room Management [J]. Fujian Computer. 2012.01

[4] Jianhua Yuan. The Application of Desktop Virtualization Technique in University Computer Room Management [J]. Fujian Commercial College Academic Journal. 2012. 02

[5] Weining Liu. Manufacture of Combination of Multi-task oriented Cloud Service [J]. CIMS, 2013, 19(1):199-209.

[6] Zhengpeng Liu. The Application of Desktop Virtualization Technique in Laboratory Equipment Management [J]. Laboratory Science, 2011, 10(1):151-153. 
[7] Fang Han, YuBin Yuan. Approaches for Actualization Desktop Virtualization Technique in Teaching and Management [J]. Chongqing University of Technology Academic Journal, 2014, 10(7):105-109.

[8] Man Lin.The Application of VDI in College Laboratory Teaching [J]. Computer CD Software and Application, 2013, 2(18):205-206.

[9] Emerging technologies for education: the 2013 Horizon Report [EB/OL].

[10]Zhiyun Gao. Discussion and Analysis on the Effect of Mutual Teaching pattern in the College Computer Class [J]. Exam Weekly.2015

作者简介: 吴士杰 (1978一), 男, 天津市人, 讲师, 主要研究计算机信息管理及网络技术。 